\title{
Particle Filters for Joint Timing and Carrier Estimation: Improved Resampling Guidelines and Weighted Bayesian Cramér-Rao Bounds
}

\author{
Ali A. Nasir, Student Member, IEEE, Salman Durrani, Senior Member, IEEE, \\ and Rodney A. Kennedy, Fellow, IEEE
}

\begin{abstract}
This paper proposes a framework for joint blind timing and carrier offset estimation and data detection using a Sequential Importance Sampling (SIS) particle filter in Additive White Gaussian Noise (AWGN) channels. We assume baud rate sampling and model the intractable posterior probability distribution functions for sampling timing and carrier offset particles using beta distributions. To enable the SIS approach to estimate static synchronization parameters, we propose new resampling guidelines for dealing with the degeneracy problem and fine tuning the estimated values. We derive the Weighted Bayesian Cramér Rao Bound (WBCRB) for joint timing and carrier offset estimation, which takes into account the prior distribution of the estimation parameters and is an accurate lower bound for all considered Signal to Noise Ratio (SNR) values. Simulation results are presented to corroborate that the Mean Square Error (MSE) performance of the proposed algorithm is close to optimal at higher SNR values (above 20 dB). In addition, the bit error rate performance approaches that of the perfectly synchronized case for small unknown carrier offsets and any unknown timing offset. The advantage of our particle filter algorithm, compared to existing techniques, is that it can work for the full range acquisition of carrier offsets.
\end{abstract}

Index Terms-Synchronization, timing offsets, carrier offsets, particle filter, Cramér-Rao bounds.

\section{INTRODUCTION}

$\mathbf{S}$ YNCHRONIZATION is a fundamental requirement for any digital communication receiver to work properly. The receiver needs to determine the correct instants of time at which to sample the incoming signal (timing synchronization). In addition, for bandpass communications, the receiver needs to adapt the frequency of its local carrier oscillator with those of the received signal (carrier or frequency offset synchronization). The synchronization techniques in the literature can be divided into two main groups: (i) data aided schemes which use training or pilot symbols which are known to both the transmitter and the receiver [1] and (ii) blind schemes which do not require any initial training [2].

Particle filters are a very popular class of numerical methods for blindly estimating the value of an observed variable that evolves in time [3]-[5]. The core principle of particle filters is to build a recursive Bayesian filter in order to estimate the

Paper approved by E. Serpedin, the Editor for Synchronization and Sensor Networks of the IEEE Communications Society. Manuscript received September 13, 2010; revised May 2, 2011 and November 16, 2011.

The authors are with the Research School of Engineering, College of Engineering and Computer Science, The Australian National University, Australia (e-mail: \{ali.nasir, salman.durrani, rodney.kennedy\}@anu.edu.au).

Digital Object Identifier 10.1109/TCOMM.2012.022912.100559 posterior probability density function of the unknown parameters. In the last decade, there has been an increasing interest in the use of particle filtering (also known as Sequential Monte Carlo algorithms) to solve estimation problems in wireless communication systems in real-time. Traditional applications of particle filters has been in mobility tracking [6]-[8] and channel estimation [9], [10] or equalization [11], [12] for wireless communications systems. Recently, particle filters have been proposed for estimating either timing or carrier offsets in different wireless communication systems [13]-[15]. The seminal work in [13] proposed a particle filtering algorithm for estimating time-varying timing offset in a single user single antenna system. Particle filters are used for variable carrier offset estimation in [14], [15], but the proposed solutions rely on the specific structure of MIMO-OFDM systems and are therefore limited to such systems. A major limitation of the above works is that they model the synchronization parameters as time-varying to make it easy to fit into standard sequential Bayesian filtering algorithms. However, in practice, the variation of the synchronization parameters is negligible over short time intervals and synchronization parameters are assumed to be constant for the duration of a transmission frame [16], [17]. For static parameters, it is well known that the lack of dynamics in the parameters causes the particle filters to lose their ability to explore the sample space after a few iterations (particle degeneracy phenomenon) [18], [19].

The estimation of static parameters using particle filters is a long standing open problem in the literature [18], [19]. Although various modifications to particle filter methods have been proposed to deal with the degeneracy problem, none is entirely satisfactory for the synchronization problem at hand. Markov Chain Monte Carlo (MCMC) based methods add diversity among the particles to deal with the degeneracy problem but it is well known that there is an accumulation of errors over time and the algorithm can diverge [20]. The artificial dynamics approach in [21] adds a small noise term to the static parameter. However this changes the original problem and is also known to suffer from error accumulation over time. Storvik's method [22] proposes to marginalize the static parameters out of the posterior distribution. However this method assumes that the concerned parameters depend on sufficient statistics which does not apply in our case. Convolution particle filters (CPF) combine regularization with convolution kernels to deal with static parameters but the evaluation of the CPF density function is a very computationally inten- 
sive process for accurate estimation of the synchronization parameters [23]. A modified particle filter is combined with gradient algorithm in [24], but the approach is very sensitive to the initialization of different parameters. A Rao-Blackwellized Particle Filter (RBPF), cross coupled with a Kalman filter, is proposed in [25] to deal with static parameter estimation. This method has the merit that linear part of the system model is estimated by a Kalman filter and the non-linear part is estimated by a particle filter. However, in our case, static parameters cannot be factored out to formulate a linear Gaussian state space model. The design of particle filters for estimating static synchronization parameters is, therefore, a challenging research problem.

It must be mentioned that non-particle filter based blind solutions for the joint synchronization problem exist in literature. Constant Modulus Algorithm (CMA) based solutions exist separately for timing and carrier offset estimation in [26] and [27], respectively. These can be combined for joint estimation but the carrier offset estimation range is very small, typically limited to $(-0.0001,0.0001)$ cycles/sample [27]. Joint timing and carrier offset estimation has also been achieved in OFDM based systems [28], [29], but the solution exploits the cyclic prefix, i.e., specific for OFDM systems, and frequency acquisition range is limited by a factor depending on the number of sub carriers used. Recently, a complete solution for joint timing and carrier offset estimation in AWGN channels has been proposed in [2]. The solution relies on the kurtosis of the received signal, but the carrier offset estimation capability is confined to the range of $(-0.05,0.05)$ cycles/sample. Moreover, their solution requires oversampling of the received signal. To the best of our knowledge, no complete solution exists for joint blind timing and carrier offset estimation with full acquisition range.

For benchmarking the performance of any proposed estimators, bounds on the achievable synchronization accuracy of timing and carrier offsets have been derived in the literature under a variety of assumptions. Traditionally, the Cramér Rao Bound (CRB) is used as the performance benchmark for any unbiased static parameter estimator. Associated CRB's have been derived for individual timing and carrier offset estimation, and joint estimation of timing and carrier offset estimation with different assumptions [30], [31]. However, the CRB does not take prior information about the static parameter into account. The most common bound that considers the prior information is the Bayesian CRB (BCRB). However as demonstrated in [31], BCRB does not exist for parameters with uniform distribution and thus cannot be used in our case. The Posterior Cramér Rao Bound (PCRB) is also not applicable in our case since it is the appropriate bound for time-varying parameter estimator [19]. For static parameters with a given finite range and uniform distribution, the Weighted Bayesian Cramér Rao Bound (WBCRB) was introduced in [31] and derived for the case of joint timing and channel estimation in [32]. It was shown to be a more accurate bound, compared to CRB for data aided scenarios. The derivation of the WBCRB for joint timing and carrier offset estimation is addressed in this work. The WBCRB is adopted as a lower bound for the performance of the proposed particle filter algorithm [33].

\section{A. Approach and Contribution}

In this paper, we are concerned with the design of a particle filtering based algorithm for non-data aided, joint estimation of transmitted symbols and static timing and carrier offsets in Additive White Gaussian Noise (AWGN) channels for a single user single antenna system. We adopt the Sequential Importance Sampling (SIS) approach and address the following practical design questions: How can we deal with the degeneracy issue when a SIS particle filter is used for estimating static parameters? Can we achieve full-range acquisition of normalized timing and carrier offsets with particle filter estimation, without any need for oversampling the received signal? The main contributions of this paper are summarized as follows:

- We use a Sequential Importance Sampling (SIS) particle filter for static timing and carrier offset estimation and we propose three new resampling guidelines for (i) dealing with the degeneracy problem (ii) resolving carrier offset estimation ambiguity at the edges of the frequency range and (iii) resolving similar amplitude ambiguity of timing offset estimates. These resampling steps are in addition to the standard resampling operation which is an integral part of the SIS approach. We verify experimentally that, in the context of the synchronization problem considered in this paper, these resampling guidelines successfully solve the degeneracy and ambiguity problems, add negligible complexity to the overall algorithm and enable full range acquisition of timing and carrier offsets.

- We derive the WBCRB for the general case of timing and carrier offsets uniformly distributed in the range $(-r / 2, r / 2)$ for $r \in(0,1)$. We show that at high Signal to Noise Ratio (SNR) the WBCRB converges to the CRB, while at low SNR the WBCRB goes flat and converges to the variance of the uniform distribution distribution of the timing and carrier offsets. In addition, the SNR value, at which the WBCRB converges to the CRB depends on the range of the estimation parameters. Thus, the WBCRB is an accurate and useful lower bound for all SNR values.

- Simulation results indicate that our proposed algorithm, with the new resampling guidelines, converges very fast to desirable accuracy within $15-20$ symbols. It provides excellent performance that is close to the WBCRB bound at high SNR in terms of Mean Square Error (MSE) performance even with relatively small number of particles. Furthermore, the Bit Error Rate (BER) performance approaches that of the perfectly synchronized case for small unknown carrier offsets and any unknown timing offset. The advantage of our solution, compared to existing techniques, is that it can also work for the full range acquisition of carrier offsets.

This paper is organised as follows. The signal model is provided in Section II and the particle filter formulation is given in Section III. The proposed particle filtering algorithm is explained in Section IV with the new resampling guidelines discussed in Section V. The WBCRB is derived in Section VI. The simulation results are presented in Section VII. Finally, conclusions are drawn in Section VIII. 
The following notation is used in the paper. Bold capital letters denote matrices and bold lower case letters denote vectors. $(\cdot)^{H}$ denotes hermitian (conjugate transpose) operator. $(\cdot)^{T}$ denotes transpose. $\operatorname{diag}(\cdot)$ denotes a diagonal matrix. $\mathbb{E}(\cdot)$ denotes expectation. $\{\cdot\}$ are used to indicate sequences of symbols or vectors while [.] are used to indicate vectors or matrices, and "*" is the convolution operator. Finally, $x \sim \mathcal{C N}\left(0, \sigma^{2}\right)$ indicates that $x$ follows a complex Gaussian distribution with 0 mean and variance $\sigma^{2}$.

\section{SYSTEM MODEL}

We consider a digital communication system where the transmitter sends the data symbols, chosen from a discrete alphabet of size $M$, over an Additive White Gaussian Noise (AWGN) channel. The noisy signal at the output of the receiver matched filter can be written as

$$
z(t)=e^{j 2 \pi F t} \sum_{d=0}^{D-1} x_{d} h(t-d T+\tau T)+v(t),
$$

where $x_{d}$ is the transmitted symbol at frame index $d, D$ is the frame size, $h(t)$ represents the overall pulse shaping filter response for the transmitter and the receiver, $T$ is the symbol period, $\tau$ is the normalized fractional unknown timing offset between the transmitter and receiver $\left(|\tau| \leq \frac{1}{2}\right), F$ is the carrier offset between the transmitter and receiver oscillators in $\mathrm{Hz}$, $v(t)=w(t) * h_{R}(t)$ is the filtered noise, $h_{R}(t)$ is the receiver pulse shaping filter response, and $w(t) \sim \mathcal{C N}\left(0, \sigma_{w}^{2}\right)$ is the zero-mean complex baseband AWGN with variance $\sigma_{w}^{2}$. The equivalent discrete-time system model after sampling is given by

$$
z_{k}=e^{j 2 \pi f k} \sum_{d=0}^{D-1} x_{d} h\left(k T_{s}-d T+\tau T\right)+v_{k},
$$

where $k=0,1, \ldots, D Q-1, z_{k}=z\left(k T_{s}\right), v_{k}=v\left(k T_{s}\right)$, $T_{s}=T / Q$ is the sampling period, $Q$ is the number of samples per symbol and $f=F / F_{s}$ is the digital frequency offset in cycles/sample. The carrier offset, $f$, has the range $\left(-B /\left(2 F_{s}\right), B /\left(2 F_{s}\right)\right)$ for the baseband signal model lying between $-B / 2$ and $B / 2 \mathrm{~Hz}$, where $B$ is the pass-band signal bandwidth.

Assuming $h(t)$ to be a causal filter with finite span $[0, L T]$, the sampled received signal is given by

$$
z_{k}=e^{j 2 \pi f k} \sum_{d=0}^{L Q} x_{k-L Q+d}^{\prime} h\left(L T-d T_{s}+\tau T\right)+v_{k},
$$

where $x_{p}^{\prime}$ is the upsampled version of the transmitted symbols stream with $Q-1$ zeros padded in between the transmitted symbols $x_{d}$, i.e., $x_{p}^{\prime}=x_{d}$ for $p=Q d$ and $x_{p}^{\prime}=0$ otherwise.

In this paper, we assume perfect frame synchronization which can be achieved in blind systems by exploiting higher order statistics of the received signal [2]. We sample the received signal at the baud rate and demonstrate excellent results for the proposed algorithm without oversampling, i.e., we use $Q=1$. This implies $F_{s}=B$, and thus we can achieve synchronization for the full range of carrier offset $(-0.5,0.5)$ and the timing offset $\left(|\tau| \leq \frac{1}{2}\right)$ respectively. Thus (3) can be written as

$$
z_{k}=e^{j 2 \pi f k} \sum_{d=0}^{L} x_{k-L+d} h(L T-d T+\tau T)+v_{k}
$$

where the sampled noise $v_{k}$ is white with the same statistical properties as $w_{k}$ for $Q=1$, i.e., $v_{k} \sim \mathcal{C N}\left(0, \sigma_{v}^{2}\right)$ and $\sigma_{v}^{2}=$ $\sigma_{w}^{2}$. The above equation can be written in vector form as

$$
z_{k}=\mathbf{x}_{k}^{T} \mathbf{h}(\tau) e^{j 2 \pi f k}+v_{k}
$$

where the superscript $(\cdot)^{T}$ denotes the transpose operator, $\mathbf{x}_{k}=\left[x_{k-L}, \ldots, x_{k}\right]^{T}$ and $\mathbf{h}(\tau)=[h(L T+$ $\tau T), \ldots, h(\tau T)]^{T}$ are $(L+1) \times 1$ vectors. (5) can be written in the matrix form as

$$
\mathbf{z}=\mathbf{D}(f) \mathbf{X h}(\tau)+\mathbf{v},
$$

where $\mathbf{z}=\left[z_{0}, z_{1}, \ldots, z_{D-1}\right]^{T}, \mathbf{v}=\left[v_{0}, v_{1}, \ldots, v_{D-1}\right]^{T}$, $\mathbf{X}=\left[\mathbf{x}_{0}, \mathbf{x}_{1}, \ldots, \mathbf{x}_{D-1}\right]^{T}$ and the diagonal matrix $\mathbf{D}=$ $\operatorname{diag}\left(e^{j 2 \pi f(0)}, e^{j 2 \pi f(1)}, \ldots, e^{j 2 \pi f(D-1)}\right)$.

\section{A. Estimation Objective}

The synchronization problem, in the above context, is to estimate the sequence of data symbols $\left\{x_{0}, \ldots, x_{D-1}\right\}$, static carrier offset $f$ and static timing offset $\tau$, from the available sequence of observations $\left\{z_{0}, \ldots, z_{D-1}\right\}$ only. From a Bayesian perspective, all the necessary information for the estimation of unknown parameters $\left(x_{k}, f, \tau\right)$ at time $k$, is contained in the joint posterior probability distribution function $p\left(\mathbf{x}_{0: k}, f, \tau \mid z_{0: k}\right)$. Since this distribution is analytically intractable, we use a particle filter to represent the posterior PDF by means of a discrete probability measure with random support. Note that $z_{0: k}=\left\{z_{0}, z_{1}, \ldots, z_{k}\right\}$ denotes the observed sequence at the receiver while $\mathbf{x}_{0: k}=\left\{\mathbf{x}_{0}, \mathbf{x}_{1}, \ldots, \mathbf{x}_{k}\right\}$ denotes a sequence of data vectors from time step 0 to $k$ respectively.

\section{B. State Space Representation}

The application of particle filtering technique necessitates the state space modeling of the problem. Taking into account the structure of the transmitted symbols, this can be given as

$$
\begin{gathered}
\mathbf{x}_{k}=\mathbf{S x}_{k-1}+\mathbf{d}_{k}, \quad \text { state equation, } \\
z_{k}=\mathbf{x}_{k}^{T} \mathbf{h}(\tau) e^{j 2 \pi f k}+v_{k}, \\
\text { observation equation, }
\end{gathered}
$$

where

$$
\mathbf{S}=\left[\begin{array}{ccccc}
0 & 1 & 0 & \ldots & 0 \\
0 & 0 & 1 & \ldots & 0 \\
\vdots & \vdots & \vdots & \ddots & \vdots \\
0 & 0 & 0 & \ldots & 1 \\
0 & 0 & 0 & \ldots & 0
\end{array}\right]
$$

is an $(L+1) \times(L+1)$ shifting matrix, $f$ and $\tau$ are static unknown parameters and $\mathbf{d}_{k}=\left[0, \ldots, 0, x_{k}\right]^{T}$ is the $(L+1) \times$ 1 perturbation vector that contains the new symbol $x_{k}$. 


\section{Particle Filter Formulation}

In this section, we present the particle filter formulation for the solution of the estimation objective in Section II-A. The particle filtering approach approximates the posterior pdf of unknown variables, $p\left(\mathbf{x}_{0: k}, f, \tau \mid z_{0: k}\right)$, by a set of $N$ particles, $\left(\left(\mathbf{x}_{0: k}, f_{0: k}, \tau_{0: k}\right)^{(i)}\right)_{i=1}^{N}$, with associated weights $\left(w_{k}^{(i)}\right)_{i=1}^{N}$, i.e.,

$p\left(\mathbf{x}_{0: k}, f, \tau \mid z_{0: k}\right)=\sum_{i=1}^{N} w_{k}^{(i)} \delta\left(\left(\mathbf{x}_{0: k}, f_{k}, \tau_{k}\right)^{(i)}-\left(\mathbf{x}_{0: k}, f, \tau\right)\right)$,

where $\delta(\cdot)$ denotes Dirac's delta function. Note that $f$ and $\tau$ denote the static unknown carrier and timing offset respectively while $f_{0: k}^{(i)}$ and $\tau_{0: k}^{(i)}$ denote the particles. The subscripts $0: k$ with the particles distinguish among the particles at each time step since new particles are generated for every received symbol depending on the weight distribution of previous particles.

In this work, we employ the Sequential Importance Sampling (SIS) particle filter algorithm [34] to determine the weights. The basic principle of the SIS algorithm is to build a recursive empirical approximation of a desired pdf by drawing samples from a different distribution called the importance function $\pi(\cdot)$ and assigning appropriate normalised weights to these samples. The choice of the importance function affects the variance of the estimates. The condition on the choice of the importance function is that it must be strictly positive and have the same domain as the desired pdf which is to be estimated [13]. The SIS algorithm has the following main steps:

1) Initialization: The algorithm is initialized using the prior distribution of the unknown variables.

2) Importance sampling: The particles are drawn according to the chosen importance function.

3) Weight update: The resulting particles are assigned weights of the form [13]

$$
w_{k}^{(i)}=\frac{p\left(\mathbf{x}_{0: k}^{(i)}, f_{0: k}^{(i)}, \tau_{0: k}^{(i)} \mid z_{0: k}\right)}{\pi\left(\mathbf{x}_{0: k}^{(i)}, f_{0: k}^{(i)}, \tau_{0: k}^{(i)} \mid z_{0: k}\right)} .
$$

4) Resampling: Resampling of the particles is required to deal with the degeneracy phenomenon, i.e., after several recursions only a few particles have non-negligible weight. Resampling redistributes the particles such that there are higher concentrations in the region where the original particles have higher weights.

5) Estimation: Using (8) and (9), the maximum a posteriori (MAP) estimates of unknown variables are calculated.

Since the posterior pdf of unknown variables is $p\left(\mathbf{x}_{0: k}, f_{0: k}, \tau_{0: k} \mid z_{0: k}\right)$, an importance function of the form $\pi\left(\mathbf{x}_{0: k}, f_{0: k}, \tau_{0: k} \mid z_{0: k}\right)$ is required. We choose an importance function that admits a factorization containing the three posterior pdf's of the variables to be estimated, i.e.,

$$
\begin{aligned}
& \pi\left(\mathbf{x}_{0: k}^{(i)}, f_{0: k}^{(i)}, \tau_{0: k}^{(i)} \mid z_{0: k}\right) \\
&=\left(\mathbf{x}_{k}^{(i)}, f_{k}^{(i)}, \tau_{k}^{(i)} \mid \mathbf{x}_{0: k-1}^{(i)}, f_{0: k-1}^{(i)}, \tau_{0: k-1}^{(i)}, z_{0: k}\right) \\
& \quad \times \pi\left(\mathbf{x}_{0: k-1}^{(i)}, f_{0: k-1}^{(i)}, \tau_{0: k-1}^{(i)} \mid z_{0: k-1}\right) \\
&=p\left(\mathbf{x}_{k}^{(i)} \mid \mathbf{x}_{0: k-1}^{(i)}, f_{0: k}^{(i)}, \tau_{0: k}^{(i)}, z_{0: k}\right) \\
& \quad \quad p\left(f_{k}^{(i)} \mid f_{0: k-1}^{(i)}, \tau_{0: k-1}^{(i)}, \mathbf{x}_{0: k-1}^{(i)}, z_{0: k-1}\right) \\
& \quad \times p\left(\tau_{k}^{(i)} \mid \tau_{0: k-1}^{(i)}, f_{0: k-1}^{(i)}, \mathbf{x}_{0: k-1}^{(i)}, z_{0: k-1}\right) \\
& \quad \times \pi\left(\mathbf{x}_{0: k-1}^{(i)}, f_{0: k-1}^{(i)}, \tau_{0: k-1}^{(i)} \mid z_{0: k-1}\right) .
\end{aligned}
$$

Using Bayes theorem, the posterior pdf of unknown variables $p\left(\mathbf{x}_{0: k}, f_{0: k}, \tau_{0: k} \mid z_{0: k}\right)$ can be written as

$$
\begin{aligned}
& p\left(\mathbf{x}_{0: k}^{(i)}, f_{0: k}^{(i)}, \tau_{0: k}^{(i)} \mid z_{0: k}\right) \\
& =\frac{p\left(\mathbf{x}_{0: k}^{(i)}, f_{0: k}^{(i)}, \tau_{0: k}^{(i)}, z_{0: k}\right)}{p\left(z_{0: k}\right)} \\
& =\frac{p\left(\mathbf{x}_{0: k}^{(i)}, f_{0: k}^{(i)}, \tau_{0: k}^{(i)}, z_{k}, z_{0: k-1}\right)}{p\left(z_{k}, z_{0: k-1}\right)} \\
& =\frac{p\left(\mathbf{x}_{0: k}^{(i)}, f_{0: k}^{(i)}, \tau_{0: k}^{(i)}, z_{k} \mid z_{0: k-1}\right) p\left(z_{0: k-1}\right)}{p\left(z_{k} \mid z_{0: k-1}\right) p\left(z_{0: k-1}\right)} \\
& =C_{k} \times p\left(\mathbf{x}_{0: k}^{(i)}, f_{0: k}^{(i)}, \tau_{0: k}^{(i)}, z_{k} \mid z_{0: k-1}\right),
\end{aligned}
$$

where $C_{k}=p\left(z_{k} \mid z_{0: k-1}\right)^{-1}$ is a normalising constant. Following the derivation in Appendix A, (11) can be further expanded to obtain a form which contains three of the pdfs in (10) as

$$
\begin{aligned}
p\left(\mathbf{x}_{0: k}^{(i)}, f_{0: k}^{(i)}\right. & \left., \tau_{0: k}^{(i)} \mid z_{0: k}\right) \\
=C_{k} & \times p\left(\mathbf{x}_{k}^{(i)} \mid \mathbf{x}_{0: k-1}^{(i)}, f_{0: k}^{(i)}, \tau_{0: k}^{(i)}, z_{0: k}\right) \\
& \times p\left(z_{k} \mid \mathbf{x}_{k-1}^{(i)}, f_{k}^{(i)}, \tau_{k}^{(i)}\right) \\
& \times p\left(\mathbf{x}_{0: k-1}^{(i)}, f_{0: k-1}^{(i)}, \tau_{0: k-1}^{(i)} \mid z_{0: k-1}\right) \\
& \times p\left(f_{k}^{(i)} \mid f_{0: k-1}^{(i)}, \tau_{0: k-1}^{(i)}, \mathbf{x}_{0: k-1}^{(i)}, z_{0: k-1}\right) \\
& \times p\left(\tau_{k}^{(i)} \mid \tau_{0: k-1}^{(i)}, f_{0: k-1}^{(i)}, \mathbf{x}_{0: k-1}^{(i)}, z_{0: k-1}\right) .
\end{aligned}
$$

Substituting (12) and (10) in (9) and simplifying, we obtain

$$
w_{k}^{(i)}=C_{k} w_{k-1}^{(i)} p\left(z_{k} \mid \mathbf{x}_{k-1}^{(i)}, f_{k}^{(i)}, \tau_{k}^{(i)}\right) .
$$

We can see that three pdfs in (10) need to be evaluated to accomplish importance sampling and a fourth pdf in (13) needs to be evaluated for weight update. The techniques for evaluation of these pdfs and the detailed implementation steps of the proposed SIS algorithm are explained in the next section.

\section{Proposed SIS Algorithm}

In this section, we describe the proposed SIS algorithm for joint data detection and frequency and timing offset estimation. The detailed implementation steps of the algorithm are as follows:

\section{A. Initialization}

For initialization, we need to determine the prior distribution of the transmitted symbol and frequency and timing offset, i.e., $p\left(x_{-1}, f_{-1}, \tau_{-1}\right)$. We assume that the transmitted symbol, frequency offset and timing offset are all independent of each 
other, i.e., $p\left(x_{-1}, f_{-1}, \tau_{-1}\right)=p\left(x_{-1}\right) p\left(f_{-1}\right) p\left(\tau_{-1}\right)$. Using the fact that modulation symbols are equally likely, we have $p\left(x_{-1}\right)=1 / M$. Similarly $p\left(f_{-1}\right)=p\left(\tau_{-1}\right)=1$ since the frequency offset and the timing offset are considered to be uniformly distributed in the range $(-0.5,0.5)$. In addition, we assume that the first symbol, $x_{-1}$, is known. This assumption helps us to avoid the $M$-fold ambiguity of the constellation and to acquire the full-range acquisition of the frequency offset. Note that we can avoid this assumption if we use differential modulation. However, the use of a single pilot symbol to resolve the ambiguities inherent in blind estimation is a common approach [35], [36]. Thus, we initialize the algorithm at time $k=-1$ as $x_{-1}^{(i)}=x_{-1}, f_{-1}^{(i)} \sim \mathcal{U}(-0.5,0.5)$ and $\tau_{-1}^{(i)} \sim \mathcal{U}(-0.5,0.5), i=1,2, \ldots, N$, where $\mathcal{U}(a, b)$ denotes the Uniform distribution from $a$ to $b$. We initialize the weights of all the particles to be equal, i.e., $w_{-1}^{(i)}=1 / N$.

\section{B. Importance Sampling}

The sampling from the importance function in (10) is accomplished in two steps. First, we need to obtain the frequency offset sample at $k^{\text {th }}$ time step from $p\left(f_{k}^{(i)} \mid f_{0: k-1}^{(i)}, \tau_{0: k-1}^{(i)}, \mathbf{x}_{0: k-1}^{(i)}, z_{0: k-1}\right) \quad$ and the timing offset sample at $k^{\text {th }}$ time step from $p\left(\tau_{k}^{(i)} \mid \tau_{0: k-1}^{(i)}, f_{0: k-1}^{(i)}, \mathbf{x}_{0: k-1}^{(i)}, z_{0: k-1}\right)$. Since these densities cannot be exactly determined, we must approximate them using a suitable density. Following the approach in [37], we approximate these distributions by a beta distribution. Since beta distribution has a range $[0,1]$, we draw the frequency and timing offset sample from the beta distribution as $\mathfrak{f}_{k} \sim \beta\left(\mathfrak{f}_{k} ; U_{k}, V_{k}\right)$ and $\zeta_{k} \sim \beta\left(\zeta_{k} ; Y_{k}, Z_{k}\right)$, where $\mathfrak{f}=f+0.5$ and $\zeta=\tau+0.5$, $\beta(y, a, b)=\int_{0}^{1} y^{a-1}(1-y)^{b-1} d y$ is the beta function and beta distribution parameters $U_{k}, V_{k}, Y_{k}$ and $Z_{k}$, are obtained by [37], such as $U_{k}=\overline{\mathfrak{f}}_{k}\left(\left(\overline{\mathfrak{f}}_{k}\left(1-\overline{\mathfrak{f}}_{k}\right) / \sigma_{\mathfrak{f}_{k}}^{2}\right)-1\right), V_{k}=$ $\left(1-\overline{\mathfrak{f}}_{k}\right)\left(\left(\overline{\mathfrak{f}}_{k}\left(1-\overline{\mathfrak{f}}_{k}\right) / \sigma_{\mathfrak{f}_{k}}^{2}\right)-1\right), Y_{k}=\bar{\zeta}_{k}\left(\left(\bar{\zeta}_{k}\left(1-\bar{\zeta}_{k}\right) / \sigma_{\zeta_{k}}^{2}\right)-1\right)$ and $Z_{k}=\left(1-\bar{\zeta}_{k}\right)\left(\left(\bar{\zeta}_{k}\left(1-\bar{\zeta}_{k}\right) / \sigma_{\zeta_{k}}^{2}\right)-1\right)$ with $\overline{\mathfrak{f}}_{k}=\sum_{i=1}^{N} w_{k-1}^{(i)} \mathfrak{f}_{k-1}^{(i)}, \quad \sigma_{\mathfrak{f}_{k}}^{2}=\sum_{i=1}^{N} w_{k-1}^{(i)}\left(\mathfrak{f}_{k-1}^{(i)}-\overline{\mathfrak{f}}_{k}\right)^{2}$, $\bar{\zeta}_{k}=\sum_{i=1}^{N} w_{k-1}^{(i)} \zeta_{k-1}^{(i)}$ and $\sigma_{\zeta_{k}}^{2}=\sum_{i=1}^{N} w_{k-1}^{(i)}\left(\zeta_{k-1}^{(i)}-\bar{\zeta}_{k}\right)^{2}$.

Next, we need to draw a sample of the transmitted symbols from the distribution $p\left(\mathbf{x}_{k}^{(i)} \mid \mathbf{x}_{0: k-1}^{(i)}, f_{0: k}^{(i)}, \tau_{0: k}^{(i)}, z_{0: k}\right)$. Since the transmitted symbols are independent and identically distributed (i.i.d.) discrete uniform random variables, we can ignore the dependency on all of the previous time states and draw the new data symbol using the distribution

$$
\begin{aligned}
& p\left(x_{k}^{(i)} \mid \mathbf{x}_{0: k-1}^{(i)}, f_{0: k}^{(i)}, \tau_{0: k}^{(i)}, z_{0: k}\right) \\
& \quad=p\left(x_{k}^{(i)} \mid \mathbf{x}_{k-1}^{(i)}, f_{k}^{(i)}, \tau_{k}^{(i)}, z_{k}\right) \\
& \quad=p\left(x_{k}^{(i)}=X_{m} \mid \mathbf{x}_{k-1}^{(i)}, f_{k}^{(i)}, \tau_{k}^{(i)}, z_{k}\right) \\
& \quad \propto p\left(z_{k} \mid x_{k}^{(i)}=X_{m}, \mathbf{x}_{k-1}^{(i)}, f_{k}^{(i)}, \tau_{k}^{(i)}\right)=\mathcal{N}\left(\mu_{k}^{(i)}\left(X_{m}\right), \sigma_{v}^{2}\right),
\end{aligned}
$$

where $\mathcal{N}\left(\mu, \sigma^{2}\right)$ denotes the Gaussian distribution with mean $\mu$ and variance $\sigma^{2}, X_{m}$ is one of the possible transmitted symbols of the modulation alphabet, $\mathcal{X}=\left\{X_{1}, X_{2}, \ldots, X_{M}\right\}$ and $\mu_{k}^{(i)}\left(X_{m}\right)=\mathfrak{x}_{k}^{(i) T} \mathbf{h}\left(\tau_{k}^{(i)}\right) e^{j 2 \pi f_{k}^{(i)} k}$, where $\mathfrak{x}_{k}^{(i)}=$ $\left[x_{k-L}^{(i)}, \ldots, x_{k-1}^{(i)}, x_{k}^{(i)}=X_{m}\right]^{T}, f_{k}^{(i)}=\mathfrak{f}_{k}^{(i)}-0.5$ and $\tau_{k}^{(i)}=$ $\zeta_{k}^{(i)}-0.5$. Note that, given $\mathbf{x}_{k-1}^{(i)}$, we only need to draw the new symbol, $x_{k}^{(i)}=X_{m}$, in order to build $\mathbf{x}_{k}^{(i)}$. Using (14), we can write the probability mass function for each symbol of $\mathcal{X}$ as

$$
\rho^{(i)}\left(x_{k}=X_{m}\right)=\frac{\mathcal{N}\left(\mu_{k}^{(i)}\left(X_{m}\right), \sigma_{v}^{2}\right)}{\sum_{X \in \mathcal{X}} \mathcal{N}\left(\mu_{k}^{(i)}(X), \sigma_{v}^{2}\right)},
$$

where $i=1,2, \ldots, N$. Hence, we draw the transmitted symbol according to $x_{k}^{(i)} \sim \rho^{(i)}\left(x_{k}\right)$ and build $\mathbf{x}_{k}^{(i)}=$ $\left[x_{k-L}^{(i)}, \ldots, x_{k}^{(i)}\right]$.

\section{Weight update}

After obtaining the new particles, we update their corresponding importance weights. Since all the possible transmitted symbols are equiprobable and independent of each other, i.e., $p\left(x_{k} \mid \mathbf{x}_{k-1}^{(i)}\right)=p\left(x_{k}\right)$, we can rewrite the weight update expression in (13) by ignoring the constant factor $C_{k}$ as

$$
\begin{aligned}
\tilde{w}_{k}^{(i)} & \propto w_{k-1}^{(i)} \sum_{X \in \mathcal{X}} p\left(z_{k} \mid x_{k}=X, \mathbf{x}_{k-1}^{(i)}, f_{k}^{(i)}, \tau_{k}^{(i)}\right) \\
& =w_{k-1}^{(i)} \sum_{X \in \mathcal{X}} \mathcal{N}\left(\mu_{k}^{(i)}(X), \sigma_{v}^{2}\right),
\end{aligned}
$$

where $\tilde{w}_{k}^{(i)}$ is the non-normalized importance weight for the particle $i$. Note that for non-equiprobable transmission, weight updates can be obtained from the modified expression $w_{k-1}^{(i)} \sum_{X \in \mathcal{X}} \mathcal{N}\left(\mu_{k}^{(i)}(X), \sigma_{v}^{2}\right) p\left(x_{k}=X\right)$, where $p\left(x_{k}=X\right)$ refers to the probability of transmitted symbol being $x_{k}=X$. Finally, we normalize the weights as $w_{k}^{(i)}=\tilde{w}_{k}^{(i)} / \sum_{n=1}^{N} \tilde{w}_{k}^{(n)}$.

\section{Resampling}

A well known problem in the practical implementation of the Sequential Importance Sampling algorithm is that the discrete measure of the particles $\left(\mathbf{x}_{k}, f_{k}, \tau_{k}\right)^{(i)}$ degenerates quickly, i.e., after a few time steps, most of the importance weights have negligible values $\left(w_{k}^{(i)} \simeq 0\right)$. The solution to this problem is to resample the particles [34]. The basic resampling operation involves generation of $N$ new particles by sampling the discrete set $\left(\left(\mathbf{x}_{k}, f_{k}, \tau_{k}\right)^{(i)}\right)_{i=1}^{N}$ with probabilities $w_{k}^{(i)}$ and then resetting the importance weights to equal values $1 / N$. Note that resampling at every time step is not needed. In our algorithm, resampling is carried out whenever the effective sample size of the particle filter $N_{\text {eff }}=1 / \sum_{i=1}^{N}\left(w_{k}^{(i)}\right)^{2}$ goes below a threshold value of $N / 2$ [38]. In addition to the standard resampling as described above, we propose new resampling guidelines which are detailed in Section V.

\section{E. Estimation}

The importance weights and the drawn samples for the frequency and timing offset are used to compute the Minimum Mean Square Error (MMSE) estimate of the true carrier and timing offset as

$$
\begin{aligned}
& \hat{f}_{k}=\sum_{i=1}^{N} f_{k}^{(i)} w_{k}^{(i)}, \\
& \hat{\tau}_{k}=\sum_{i=1}^{N} \tau_{k}^{(i)} w_{k}^{(i)},
\end{aligned}
$$


TABLE I

Proposed Sequential IMPoRTANCE SAMPLING (SIS) PARTICLE FILTER ALGORITHM.

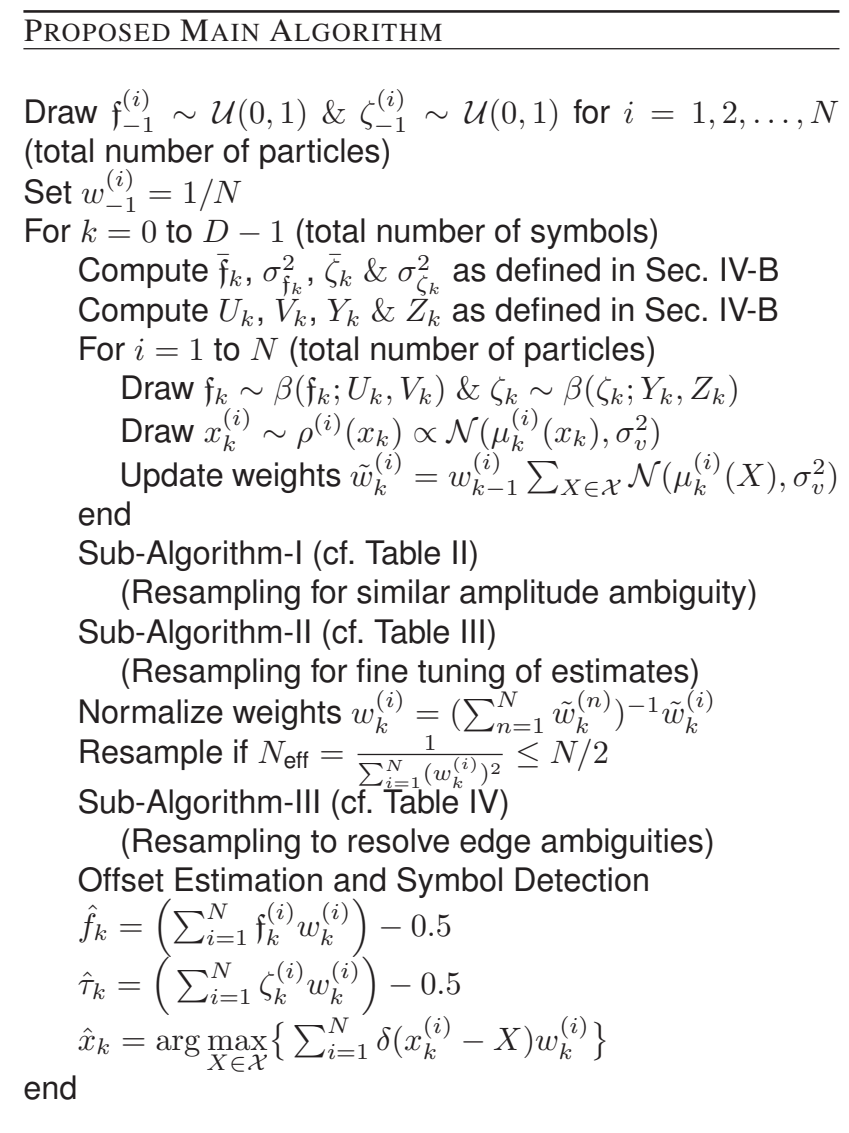

where $\hat{f}_{k}$ and $\hat{\tau}_{k}$ are MMSE estimates of true frequency and timing offsets at $k^{\text {th }}$ time step, respectively.

Finally, we compute the Maximum A Posteriori (MAP) estimates of the transmitted symbol $k$ as

$$
\hat{x}_{k}=\arg \max _{X \in \mathcal{X}}\left(\sum_{i=1}^{N} w_{k}^{(i)} \delta\left(x_{k}^{(i)}-X\right)\right) .
$$

The proposed algorithm is summarized in Table I.

\section{New Resampling Guidelines}

In this section, we propose new resampling guidelines for (i) dealing with the degeneracy problem, (ii) resolving carrier offset estimation ambiguity at the edges of the frequency range and (iii) resolving similar amplitude ambiguity of timing offset estimates. In addition, we experimentally verify that the additional computational complexity introduced as a result of these new resampling guidelines is negligible.

\section{A. Resampling for resolving similar amplitude ambiguity of timing offset estimates}

The SIS algorithm with the standard resampling operation alone may not lead to meaningful results for the timing offsets if measures to combat the similar amplitude ambiguity of timing offset estimates are not taken. Since we assume perfect frame synchronization and $\left\{x_{-L}, x_{-L+1}, \ldots, x_{-1}\right\}=0$, the pulse shaping function (raised cosine) has the same amplitude
TABLE II

SUB-ALGORITHM FOR RESOLVING SIMILAR AMPLITUDE AMBIGUITY OF TIMING OFFSET ESTIMATES

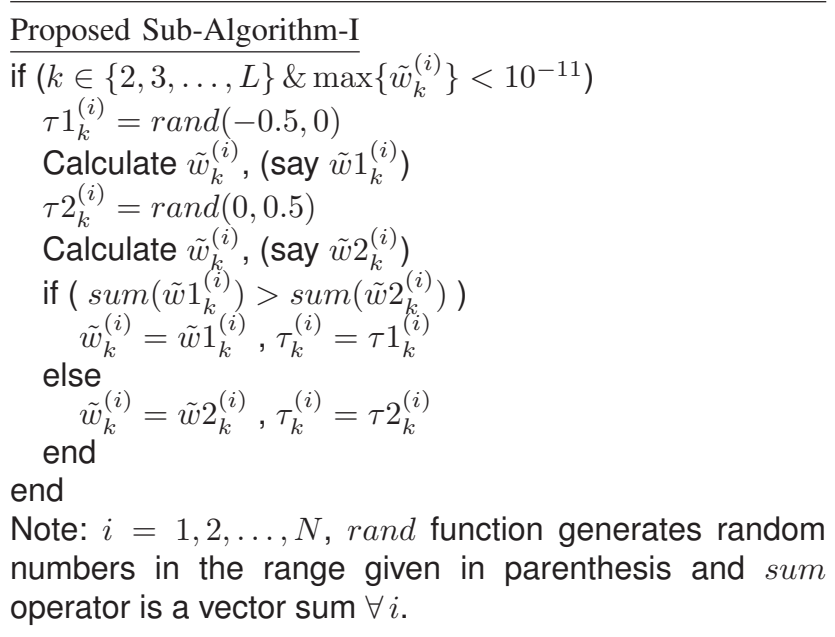

values for both positive and negative values of the timing offset [37]. Thus, the timing offsets $\tau_{-1}^{(i)}$ and $-\tau_{-1}^{(i)}$ will result in the same importance weights. Thus there is a $50 \%$ chance that the the timing offset pdf will evolve in wrong region after the first symbol.

To resolve this similar amplitude ambiguity in the timing offset, we use the fact that as more data symbols come and the sequence $\left\{x_{k-L}, x_{k-L+1}, \ldots, x_{k-1}\right\}$ start taking reasonable values, the convolution of the causal pulse shaping filter with the input symbols will help to differentiate between the corresponding positive and negative timing offset particles. Thus from second till $L+1$ symbols, where $L$ denotes the length of pulse shaping filter, we generate timing offset particles first on one side $(-0.5,0)$ and then on the other side $(0,0.5)$ of the estimation range and check the nonnormalized weights for both cases. Finally, we resample on the basis of the particles of that region which result in a greater value for the sum of non-normalized weights. This helps the particles to evolve in the correct region from the second symbol onwards. This resampling guideline is invoked only if degeneracy phenomenon is detected, i.e., the maximum weight falls below a threshold value of $10^{-11}$. This threshold value is chosen as a tradeoff to avoid incorrect symbol detection due to wrong convergence (more probable for lesser thresholds) and unnecessary premature call for the resampling guideline that can also lead to the evolution of the pdf towards the wrong region (more probable for higher thresholds). The proposed sub-algorithm-I is summarised in Table II.

\section{B. Resampling for dealing with the degeneracy problem}

To deal with the degeneracy problem arising from fixed parameter estimation with SIS particle filters, we propose a resampling guideline on the weights for both the timing and the carrier offset. In this paper, we consider the full timing and carrier offset acquisition range $(-0.5,0.5)$ as a cyclic range. If the weights fall below a selected threshold, we regenerate the particles in the range \pm 0.01 of the most recurring particle and update the corresponding weights. That is, we explore the particle space in the vicinity of the converged value to 
TABLE III

SUB-ALGORITHM FOR FINE TUNING OF TIMING AND FREQUENCY OFFSET ESTIMATES

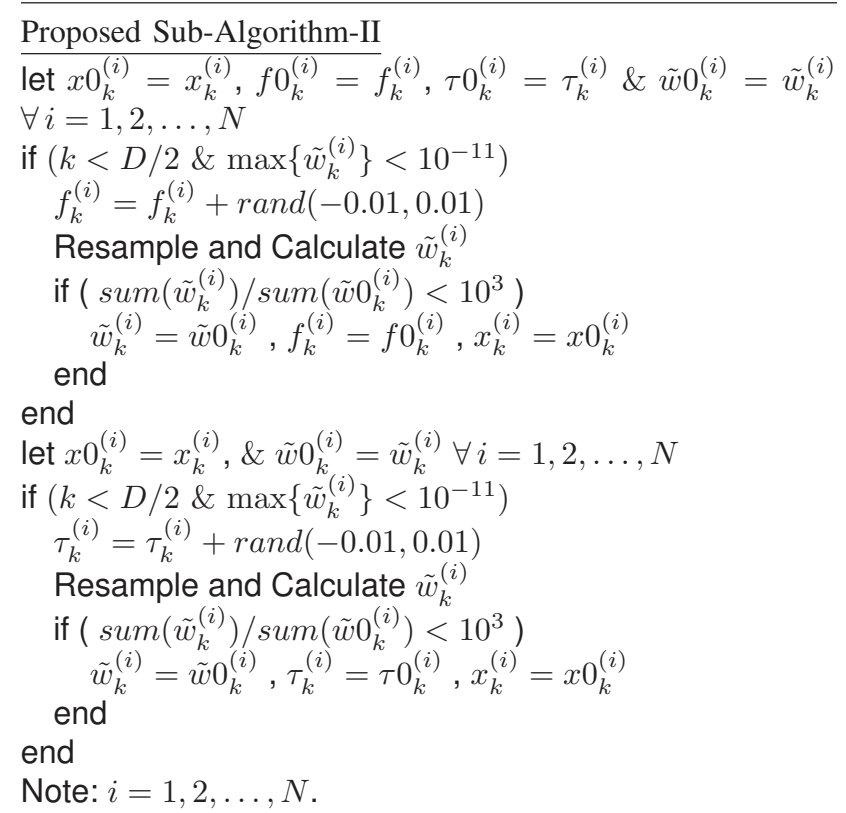

enable the algorithm to fine tune in the convergence region. We do this space exploration for carrier offset particles first since even a very small residual error in carrier offset estimation can accumulate with time and lead to failure in data detection. Then we recheck the weights and if they again fall below the selected threshold, we repeat the proposed space exploration for timing particles. The proposed sub-algorithmII is summarized in Table III.

\section{Resampling for resolving edge ambiguities of carrier offset estimates}

The edges of the carrier offset range, $(-0.5,-0.45)$ and $(0.45,0.5)$, are very sensitive to error since even a small amount of noise can perturb the received signal and lead to the posterior density of carrier offset to evolve in the wrong estimation region. We mitigate this problem by putting a check on the first symbol $(k=0)$ after resampling, i.e., if more than $90 \%$ particles are populated towards edge regions, we gather all the particles towards that end of the estimation range, which is more heavily occupied with the particles. The proposed subalgorithm-III is summarized in Table IV.

\section{Complexity Analysis}

In this subsection, we experimentally verify that the proposed resampling guidelines are only invoked under certain special conditions and they do not increase the overall computational complexity of the main algorithm in Table I. The subalgorithm-III is only invoked for the first symbol in a frame and only when more than $90 \%$ of the particles are populated towards the edge regions. The sub-algorithm-I and II are executed when particle filter degenerates. We use simulations to test the average number of times Sub-Algorithm-I and II are executed in a frame of 100 symbols. The results are summarized in Table $\mathrm{V}$ after averaging over 600 randomly
TABLE IV

SUB-ALGORITHM FOR RESOLVING EDGE AMBIGUITIES OF CARRIER OFFSET ESTIMATES

Proposed Sub-Algorithm-III

let $\epsilon=$ be most recurring frequency particle, $f_{k}^{(i)}$, among all $i=1,2, \ldots, N$. let $f_{k}^{(i)}(x, y)$ be the frequency particles that lie in the range $(x, y)$ and $\mathfrak{n}(x, y)$ be the number of those particles.

$$
\begin{aligned}
& \text { if }(\mathfrak{n}(-0.5,-0.4)+\mathfrak{n}(0.4,0.5)>0.9 N) \\
& \text { if }(\mathfrak{n}(-0.5,-0.4)>\mathfrak{n}(0.4,0.5)) \\
& \quad f_{k}^{(i)}(0.4,0.5)=\epsilon+\operatorname{rand}(-0.015,0.015) \\
& \text { else } \\
& \quad f_{k}^{(i)}(-0.5,-0.4)=\epsilon+\operatorname{rand}(-0.015,0.015) \\
& \text { end } \\
& \text { if }\left(f_{k}^{(i)}>0.5 \| f_{k}^{(i)}<0.5\right) \\
& \quad f_{k}^{(i)}=\epsilon \\
& \text { end } \\
& \text { end } \\
& \text { Note: } i=1,2, \ldots, N .
\end{aligned}
$$

TABLE V

AVERAGE NUMBER OF TIMES SUB-ALGORITHMS I AND II ARE INVOKED IN A FRAME OF 100 SYMBOLS.

\begin{tabular}{c|c|c|c}
\hline \multirow{2}{*}{ No. of Particles } & \multicolumn{3}{|c}{ Average Number of Times } \\
& SNR=20 & SNR $=24$ & SNR $=28$ \\
\hline$N=100$ & 0.098 & 0.48 & 0.88 \\
\hline$N=200$ & 0.023 & 0.053 & 0.50 \\
\hline$N=300$ & 0 & 0.01 & 0.29 \\
\hline
\end{tabular}

generated frames and random timing and frequency offsets for $N=100,200$ and 300 particles at SNR $=20,24$ and 28 $\mathrm{dB}$, respectively. The results in Table $\mathrm{V}$ show that overall the sub-algorithms-I and II are not called more than once in a frame, thus adding negligible complexity to the main particle filter algorithm.

\section{Weighted BAyesian CRAmér RaO Bounds}

In this section, we derive the estimation performance bounds. Since the derivation of CRB provides the theoretical foundation for the derivation of WBCRB, we first derive the CRB for joint timing and carrier offset estimation without assuming any prior information. This is then used to derive the WBCRB which incorporates the prior information on the range of the timing and the carrier offsets, respectively. In the WBCRB derivation, we consider the general case of timing and carrier offsets uniformly distributed in the range $(-r / 2, r / 2)$ for $r \in(0,1)$. Note that non-data aided bounds, treating transmitted data symbols as unknown vector, are beyond the scope of this paper.

\section{A. Joint $C R B$}

We start from the signal model given in (6) as $\mathbf{z}=$ $\mathbf{D X h}+\mathbf{v}$. Let $\boldsymbol{\theta} \triangleq[\tau, f]^{T}$ and denote $\boldsymbol{\mu}=\mathbf{D X h}$. Since, the covariance matrix $\mathbf{C}_{\mathbf{z}}$ does not depend on $\boldsymbol{\theta}$, the Fisher Information matrix (FIM) $\mathbf{J}$ of $\boldsymbol{\theta}$ is given by [39]

$$
\mathbf{J}(\boldsymbol{\theta})=2 \Re\left(\frac{\partial \boldsymbol{\mu}^{H}}{\partial \boldsymbol{\theta}} \mathbf{C}_{\mathbf{z}}^{-1} \frac{\partial \boldsymbol{\mu}}{\partial \boldsymbol{\theta}^{T}}\right),
$$


where $\frac{\partial}{\partial a}(\cdot)$ denotes the partial derivative with respect to $a$, the inverse covariance matrix, $\mathbf{C}_{\mathbf{z}}^{-1}=\frac{1}{\sigma^{2}} \mathbf{I}_{D}$ for the signal model in (6) and $\mathbf{I}_{D}$ is identity matrix of $D \times D$ size. Substituting in (20), we get

$$
\begin{aligned}
\mathbf{J}(\boldsymbol{\theta}) & =\frac{2}{\sigma_{v}^{2}} \Re\left(\frac{\partial \boldsymbol{\mu}^{H}}{\partial \boldsymbol{\theta}} \frac{\partial \boldsymbol{\mu}}{\partial \boldsymbol{\theta}^{T}}\right) \\
& =\frac{2}{\sigma_{v}^{2}} \Re\left(\left[\begin{array}{cc}
\frac{\partial \boldsymbol{\mu}^{H}}{\partial \tau} \frac{\partial \boldsymbol{\mu}}{\partial \tau} & \frac{\partial \boldsymbol{\mu}^{H}}{\partial \tau} \frac{\partial \boldsymbol{\mu}}{\partial f} \\
\frac{\partial \boldsymbol{\mu}^{H}}{\partial \tau} \frac{\partial \boldsymbol{\mu}}{\partial \tau} & \frac{\partial \boldsymbol{\mu}^{H}}{\partial \tau} \frac{\partial \boldsymbol{\mu}}{\partial f}
\end{array}\right]\right) .
\end{aligned}
$$

Substituting the value of $\boldsymbol{\mu}$ in (21), we get (22) as shown at the bottom of this page, where $\mathbf{T}=2 \pi j \times \operatorname{diag}(0,1, \ldots, D-$ $1), j$ is the imaginary number defined as $j=\sqrt{-1}$ and $\mathbf{h}^{\prime}=$ $\frac{\partial \mathbf{h}}{\partial \tau}$.

Using the fact that $\mathbf{D}^{H} \mathbf{D}=\mathbf{I}_{D}$ and $\mathbf{T}^{H}=-\mathbf{T}$ as $\mathbf{T}$ is purely an imaginary matrix, (22) simplifies to

$$
\mathbf{J}(\boldsymbol{\theta})=\frac{2}{\sigma_{v}^{2}}\left[\begin{array}{cc}
\Re\left(\mathbf{h}^{\prime}{ }^{H} \mathbf{X}^{H} \mathbf{X} \mathbf{h}^{\prime}\right) & \Re\left(\mathbf{h}^{\prime} \mathbf{X}^{H} \mathbf{T X h}\right) \\
\Re\left(\mathbf{h}^{H} \mathbf{X}^{H} \mathbf{T}^{H} \mathbf{X} \mathbf{h}^{\prime}\right) & \Re\left(-\mathbf{h}^{H} \mathbf{X}^{H} \mathbf{T}^{2} \mathbf{X} \mathbf{h}\right)
\end{array}\right] .
$$

Let us write (23) as

$$
\mathbf{J}(\boldsymbol{\theta})=\frac{2}{\sigma_{v}^{2}}\left[\begin{array}{ll}
A_{11} & A_{12} \\
A_{21} & A_{22}
\end{array}\right],
$$

where $A_{i j}$ are all scalars, such that $A_{11}=\Re\left(\mathbf{h}^{\prime}{ }^{H} \mathbf{X}^{H} \mathbf{X} \mathbf{h}^{\prime}\right)$, $A_{12}=\Re\left(\mathbf{h}^{\prime}{ }^{H} \mathbf{X}^{H} \mathbf{T X h}\right), A_{21}=\Re\left(\mathbf{h}^{H} \mathbf{X}^{H} \mathbf{T}^{H} \mathbf{X} \mathbf{h}^{\prime}\right)$ and $A_{22}=\Re\left(-\mathbf{h}^{H} \mathbf{X}^{H} \mathbf{T}^{2} \mathbf{T X h}\right)$. Substituting these scalar factors in (24), we get (25) as shown at the bottom of this page.

Finally, the CRB can be found by taking inverse of FIM and is defined in (25). The CRB of timing and frequency offset can thus be written as

$$
\begin{aligned}
& \operatorname{CRB}(\tau)=\frac{\sigma_{v}^{2}}{2}\left(A_{11}-A_{12} A_{22}^{-1} A_{21}\right)^{-1}, \\
& \operatorname{CRB}(f)=\frac{\sigma_{v}^{2}}{2}\left(A_{22}-A_{21} A_{11}^{-1} A_{12}\right)^{-1} .
\end{aligned}
$$

The validity of these results is discussed in Sec. VII.

\section{B. Weighted Bayesian CRB}

The WBCRB for vector parameters is defined as [31]

$$
\begin{aligned}
& \operatorname{WBCRB}(\boldsymbol{\theta}) \\
& =\mathbb{E}_{\boldsymbol{\theta}}(\mathbf{Q}(\boldsymbol{\theta}))\left[\mathbb{E}_{\boldsymbol{\theta}}\left(\mathbf{J}_{w}(\boldsymbol{\theta})\right)+\mathbb{E}_{\boldsymbol{\theta}}\left(\mathbf{P}_{w}(\boldsymbol{\theta})\right)\right]^{-1} \mathbb{E}_{\boldsymbol{\theta}}(\mathbf{Q}(\boldsymbol{\theta})),
\end{aligned}
$$

where $\mathbf{J}_{w}(\boldsymbol{\theta})$ is the weighted FIM for the timing and frequency offset estimation, $\mathbf{P}_{w}(\boldsymbol{\theta})$ is the weighted Prior Information Matrix (PIM) and $\mathbf{Q}$ is a matrix representing the weighting functions. Following [31], these terms are defined in our case as

$$
\left[\mathbf{J}_{w}(\boldsymbol{\theta})\right]_{i j}=q\left(\theta_{i}\right) q\left(\theta_{j}\right) \mathbb{E}_{\mathbf{z} \mid \boldsymbol{\theta}}\left(\frac{\partial \ln p(\mathbf{z} \mid \boldsymbol{\theta})}{\partial \theta_{i}} \frac{\partial \ln p(\mathbf{z} \mid \boldsymbol{\theta})}{\partial \theta_{j}}\right),
$$

$$
\begin{gathered}
{\left[\mathbf{P}_{w}(\boldsymbol{\theta})\right]_{i j}=q\left(\theta_{i}\right) q\left(\theta_{j}\right) \frac{\partial \ln \left[p(\boldsymbol{\theta}) q\left(\theta_{i}\right)\right]}{\partial \theta_{i}} \frac{\partial \ln \left[p(\boldsymbol{\theta}) q\left(\theta_{j}\right)\right]}{\partial \theta_{j}},} \\
\mathbf{Q}(\boldsymbol{\theta}) \triangleq \operatorname{diag}\left(q\left(\theta_{i}\right), q\left(\theta_{j}\right)\right),
\end{gathered}
$$

where $i, j=\{1,2\}, \theta_{1} \in(-r / 2, r / 2)$ and $\theta_{2} \in(-r / 2, r / 2)$ correspond to timing and carrier offsets respectively in our case, $p(\boldsymbol{\theta})=\frac{1}{r}$ is the prior distribution of timing and carrier offsets, $q\left(\theta_{n}\right)=\left(\frac{1}{r}\right)^{2 c} \phi_{n}^{c}\left(r-\phi_{n}\right)^{c}$ for $0<\phi_{n}<r, \phi_{n}=$ $\theta_{n}+\frac{r}{2}, n=\{1,2\}$ and $c$ is the weighting index. The value of $c$ can be chosen to adjust the tightness of the WBCRB. Note that if the weighting index, $c=0$, the WBCRB in (28) has the following relationship with the CRB in (25) [31]

$$
\left.\operatorname{WBCRB}(\boldsymbol{\theta})\right|_{c=0}=\left(\mathbb{E}_{\boldsymbol{\theta}}\left\{\mathrm{CRB}^{-1}(\boldsymbol{\theta})\right\}\right)^{-1} \text {. }
$$

The calculation of the WBCRB in (28) requires the computation of three expectations, which are detailed in the following subsections.

1) Calculation of $\mathbb{E}_{\boldsymbol{\theta}}(\mathbf{Q}(\boldsymbol{\theta}))$ : Given the signal model in (6) and the weighing function in (31), the expectation $\mathbb{E}_{\boldsymbol{\theta}}(\mathbf{Q}(\boldsymbol{\theta}))$ can be obtained by extending the derivation in [31] and leads to the following result

$$
\mathbb{E}_{\boldsymbol{\theta}}(\mathbf{Q}(\boldsymbol{\theta}))=\beta(c+1, c+1) \mathbf{I}_{2},
$$

where $\beta(a, b)=\int_{0}^{1} y^{a-1}(1-y)^{b-1} d y$ denotes the beta function and $\mathbf{I}_{2}$ is a $2 \times 2$ identity matrix.

2) Calculation of $\mathbb{E}_{\boldsymbol{\theta}}\left(\mathbf{P}_{w}(\boldsymbol{\theta})\right)$ : Given the signal model in (6) and the weighting function in (31), the expectation of the weighted PIM $\mathbb{E}_{\boldsymbol{\theta}}\left(\mathbf{P}_{w}(\boldsymbol{\theta})\right)$ is given by [31]

$$
\left[\mathbb{E}_{\boldsymbol{\theta}}\left(\mathbf{P}_{w}(\boldsymbol{\theta})\right)\right]_{i j}= \begin{cases}\left(\frac{1}{r}\right)^{2} c \beta(2 c+1,2 c-1), & i=j \\ 0, & i \neq j,\end{cases}
$$

where $c$ is the weighting index and $\beta(\cdot)$ is beta function.

3) Calculation of $\mathbb{E}_{\boldsymbol{\theta}}\left(\mathbf{J}_{w}(\boldsymbol{\theta})\right)$ : Exploiting the fundamental properties of the weighted FIM, we can rewrite (29) as [39]

$$
\left[\mathbf{J}_{w}(\boldsymbol{\theta})\right]_{i j}=q\left(\theta_{i}\right) q\left(\theta_{j}\right)[\mathbf{J}(\boldsymbol{\theta})]_{i j},
$$

where $[\mathbf{J}(\boldsymbol{\theta})]_{i j}=\mathbb{E}_{\mathbf{z} \mid \boldsymbol{\theta}}\left(\frac{\partial \ln p(\mathbf{z} \mid \boldsymbol{\theta})}{\partial \theta_{i}} \frac{\partial \ln p(\mathbf{z} \mid \boldsymbol{\theta})}{\partial \theta_{j}}\right)$ is the FIM and is given by (24) in our case.

Using (24) and (35), we have a closed form expression for $\mathbf{J}_{w}(\boldsymbol{\theta})$. Note that since this expression depends on the pulse shaping filter $h(t)$, it is not possible to obtain a closed form analytical solution for $\mathbb{E}_{\boldsymbol{\theta}}\left(\mathbf{J}_{w}(\boldsymbol{\theta})\right)$. However it can easily be obtained numerically. Finally, using the results in Subsections VI-B1, VI-B2 and VI-B3, the WBCRB in (28) can be found numerically. Moreover, $\operatorname{WBCRB}(\tau)=[\operatorname{WBCRB}(\boldsymbol{\theta})]_{11}$ and $\operatorname{WBCRB}(f)=[\operatorname{WBCRB}(\boldsymbol{\theta})]_{22}$. The $\mathrm{WBCRB}$ is compared with the simulation results in the next section.

$$
\mathbf{J}(\boldsymbol{\theta})=\frac{2}{\sigma_{v}^{2}}\left[\begin{array}{cc}
\Re\left\{\mathbf{h}^{\prime}{ }^{H} \mathbf{X}^{H} \mathbf{D}^{H} \mathbf{D} \mathbf{X} \mathbf{h}^{\prime}\right\} & \Re\left\{\mathbf{h}^{\prime}{ }^{H} \mathbf{X}^{H} \mathbf{D}^{H} \mathbf{T} \mathbf{D X h}\right\} \\
\Re\left\{\mathbf{h}^{H} \mathbf{X}^{H} \mathbf{D}^{H} \mathbf{T}^{H} \mathbf{D} \mathbf{X} \mathbf{h}^{\prime}\right\} & \Re\left\{\mathbf{h}^{H} \mathbf{X}^{H} \mathbf{D}^{H} \mathbf{T}^{H} \mathbf{T D X} \mathbf{D}\right\}
\end{array}\right] .
$$

$$
\operatorname{CRB}(\boldsymbol{\theta})=[\mathbf{J}(\boldsymbol{\theta})]^{-1}=\frac{\sigma_{v}^{2}}{2}\left[\begin{array}{cc}
\left(A_{11}-A_{12} A_{22}^{-1} A_{21}\right)^{-1} & -\left(A_{11}-A_{12} A_{22}^{-1} A_{21}\right)^{-1} A_{12} A_{22}^{-1} \\
-\left(A_{22}-A_{21} A_{11}^{-1} A_{12}\right)^{-1} A_{21} A_{11}^{-1} & \left(A_{22}-A_{21} A_{11}^{-1} A_{12}\right)^{-1}
\end{array}\right] .
$$




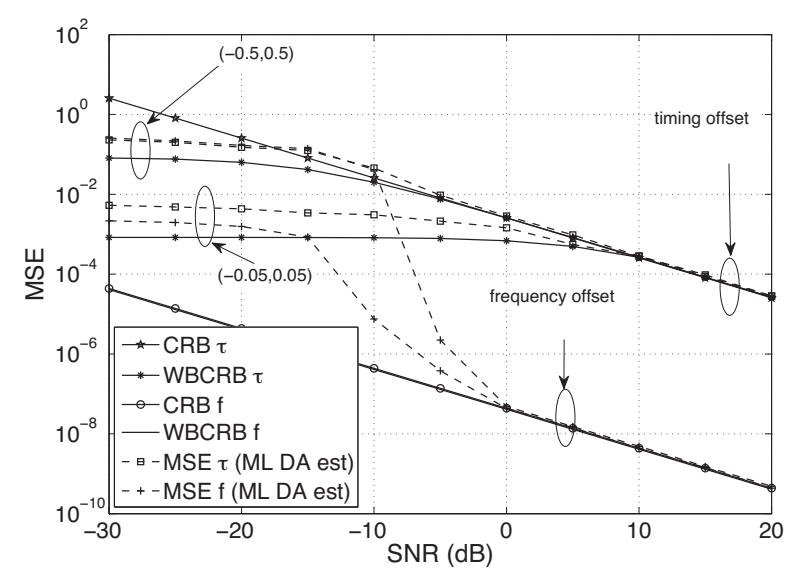

Fig. 1. CRB and WBCRB for joint timing and carrier offset estimation and MSE of timing and carrier offset estimation using optimal data-aided maximum likelihood (DA ML) estimator.

\section{Validation of $W B C R B$}

In this subsection, we show that the WBCRB provides useful additional information compared to the $\mathrm{CRB}$ and is an accurate lower bound for all SNR values. Fig. 1 plots the WBCRB in (28) for two different estimation ranges, $(-0.5,0.5)$ and $(-0.05,0.05)$, as a function of SNR. In calculating the WBCRB, the value of weighting index $c$ is set to 1 to get tightest bound [32]. Also, as discussed in Sec. VI-B3, the weighted FIM, $\mathbb{E}_{\boldsymbol{\theta}}\left\{\mathbf{J}_{w}(\boldsymbol{\theta})\right\}$, is computed numerically by averaging the results of $\mathbf{J}_{w}(\boldsymbol{\theta})$ in (35) for 10,000 simulations with randomly generated timing and carrier offsets. The CRB in (26) and (27) is the same for both estimation ranges since it does not incorporate the prior information on the range of the timing and carrier offsets.

Fig. 1 shows that at high SNR the WBCRB converges to the CRB since the estimate from the data dominates the prior information, while at low SNR the WBCRB for timing offset goes flat and converges to the variance levels $\frac{1}{12}(1)^{2}=$ 0.083 and $\frac{1}{12}(0.1)^{2}=8.3 \times 10^{-4}$ for the estimation ranges $(-0.5,0.5)$ and $(-0.05,0.05)$ respectively. For frequency offset estimation, a similar value will be reached but this requires extremely low SNR in AWGN channels. The SNR where WBCRB converges to the CRB depends on the parameter estimation range. For the full range of timing offset estimation, the WBCRB converges to the CRB at very low $\mathrm{SNR}(\approx-6$ $\mathrm{dB}$ ). However if timing offsets are uniformly distributed over a smaller range, the WBCRB converges to the CRB at mid to high SNR, e.g., at $10 \mathrm{~dB}$ for the range $(-0.05,0.05)$. Further, to illustrate the advantage of the WBCRB over the CRB, we also simulate the MSE of the timing and frequency offset estimation for the two estimation ranges using an optimal dataaided maximum likelihood (DA ML) estimator which assumes the knowledge of the transmitted data vector at the receiver. Fig. 1 shows that at low SNR, MSE of timing offset estimation is below the conventional CRB and it matches well with the WBCRB at high SNR. Thus the WBCRB is an accurate and useful lower bound for all SNR values.
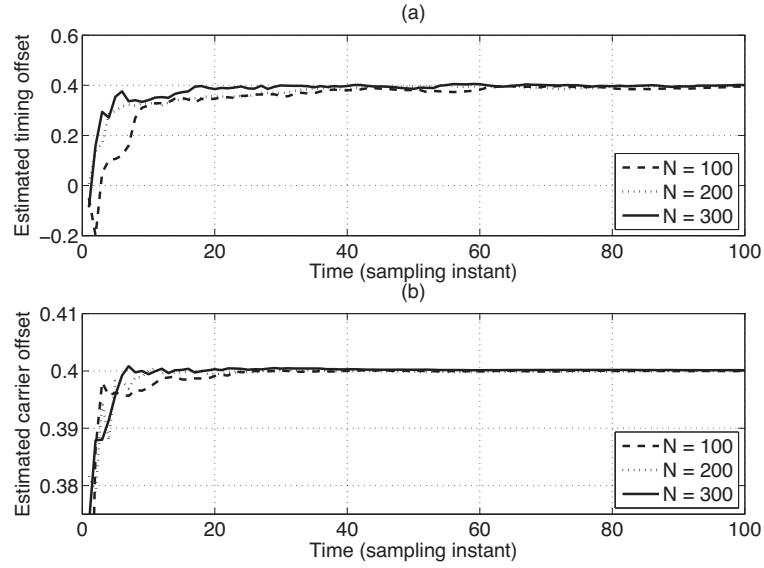

Fig. 2. Estimate of the (a) frequency offset $\hat{f}_{k}$ and (b) timing offset $\hat{\tau}_{k}$ at different time instants for SNR $=12 \mathrm{~dB}$, frequency offset $f=0.4$, timing offset $\tau=0.4$ and number of particles $N=100,200$ and 300 , respectively.

\section{Simulation Results}

In this section, we present simulation results to assess the performance of the proposed SIS particle filter algorithm. For simplicity, we use Binary Phase Shift Keying (BPSK) modulation and an AWG channel. The oversampling factor is $Q=1$. The root raised cosine causal filters are truncated to $[0,2 T]$ for transmitter pulse shaping and receiver matched filtering, with roll-off factor 0.22 and filter lag $L=2$. The timing offset is uniformly distributed in the full acquisition range $(-0.5,0.5)$, while the carrier offset is uniformly distributed in the approximately full acquisition range $(-0.48,0.48)$ for every simulation. We use $N=100,200,300$ and 1000 particles to estimate the data symbols and timing and carrier offsets jointly. All results are averaged over $R=1000$ Monte Carlo simulations, with each simulation consisting of a block of $D=100$ transmitted bits. The figures of merit used are the MSE of timing and carrier offsets and the mean BER. The MSE of timing offset is calculated as, $\operatorname{MSE}=(1 / R) \sum_{\ell=1}^{R}\left(\tau(\ell)-\hat{\tau}_{D-1}(\ell)\right)^{2}$, where $\hat{\tau}_{D-1}(\ell)$ is the estimate of timing offset at $(D-1)^{\text {th }}$ symbol and $\ell^{\text {th }}$ simulation. Similarly, the MSE of the carrier offset is calculated as, $\mathrm{MSE}=(1 / R) \sum_{\ell=1}^{R}\left(f(\ell)-\hat{f}_{D-1}(\ell)\right)^{2}$, where $\hat{f}_{D-1}(\ell)$ is the estimate of carrier offset at $(D-1)^{\text {th }}$ symbol and $\ell^{\text {th }}$ simulation. The mean BER is obtained by averaging the BER for each simulation run without discarding any initial bits.

\section{A. Performance of Proposed Particle Filter Algorithm}

Fig. 2(a) and 2(b) illustrate the convergence behaviour of the timing and carrier offset estimates respectively. The figures show the estimates of the timing and the carrier offsets at different time instants for a SNR of $12 \mathrm{~dB}$ for one particular realization of the particle filter. The true timing and carrier offset in this case is $\tau=f=0.4$, which represents a worst case scenario. We can see from Fig. 2(a) that the estimated timing offset $\hat{\tau}_{k}$ converges to the true value after about $25-30$ time instants, e.g., for $N=100$ particles, the value is $\hat{\tau}_{k}=$ 0.3588 after $k=25$ time instants which is very close to the true value. From Fig. 2(b), we can see that the estimated carrier 


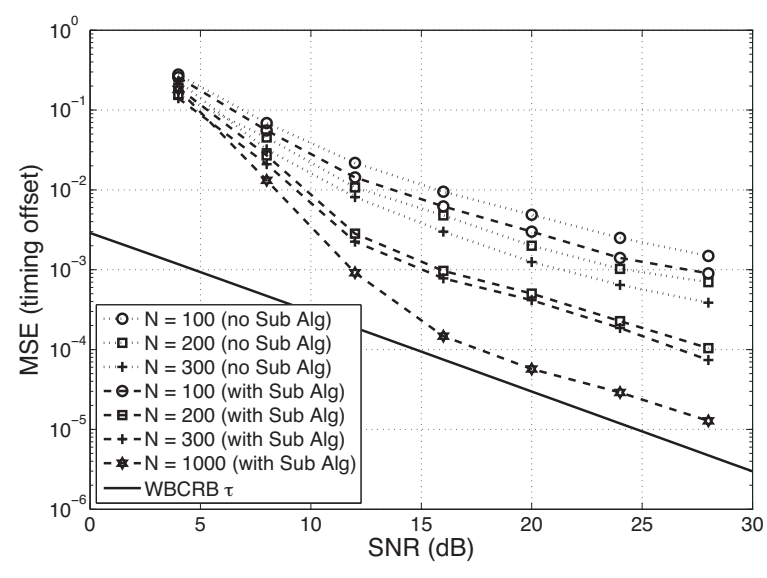

Fig. 3. MSE of timing offset estimation as a function of SNR (dB), for different number of particles $N=100,200,300$ and 1000 .

offset $\hat{f}_{k}$ converges to the true value after just $15-20$ time instants, e.g., for $N=100$ particles, the value is $\hat{f}_{k}=0.3991$ after $k=20$ time instants which is very close to the true value. In addition, as we increase the number of particles, the estimate converges more rapidly in both cases. The results in Fig. 2 exemplify the fast convergence behavior of the proposed algorithm.

Figs. 3 and 4 show the Mean Square Error (MSE) of the timing offset and the carrier offset as a function of SNR for different number of particles respectively. The WBCRB in (28) for timing and carrier offsets is also plotted as a reference. We can see that, in general, the MSE of the timing and carrier offsets decreases by increasing the SNR or increasing the number of particles. For the particle filter with new resampling guidelines, the MSE of the carrier offset estimation decreases considerably after $15 \mathrm{~dB}$ SNR and approaches the WBCRB for $N=300$ and 1000 particles. Similarly the MSE of the timing offset estimation approaches the WBCRB at high SNR for large number of particles. Comparing the curves in Figs. 3 and 4 for the cases with and without the new resampling guidelines, we can see that the new resampling guidelines proposed in Sec. V result in considerable performance improvement for both timing and carrier offset estimation and help in achieving the MSE performance close to the WBCRB at mid-to-high SNR values. Thus, this experimentally verifies that, in the context of the synchronization problem considered in this paper, these resampling guidelines successfully solve the degeneracy and ambiguity problems without adversely affecting the convergence of the SIS algorithm. In addition, we quantify the performance improvement resulting from the use of new resampling guidelines. Fig. 3 shows that for timing offset estimation, the proposed resampling guidelines attain 10 dB performance improvement for MSE of $10^{-3}$ with $N=200$ particles. Similarly, for carrier offset estimation in Fig. 4, the use of proposed resampling guidelines achieves almost $9 \mathrm{~dB}$ performance gain for MSE of $10^{-6}$ with $N=300$ particles. Note that as demonstrated in Sec. V, this performance improvement comes at the expense of negligible additional complexity.

Fig. 5 shows the mean BER of the proposed particle filter

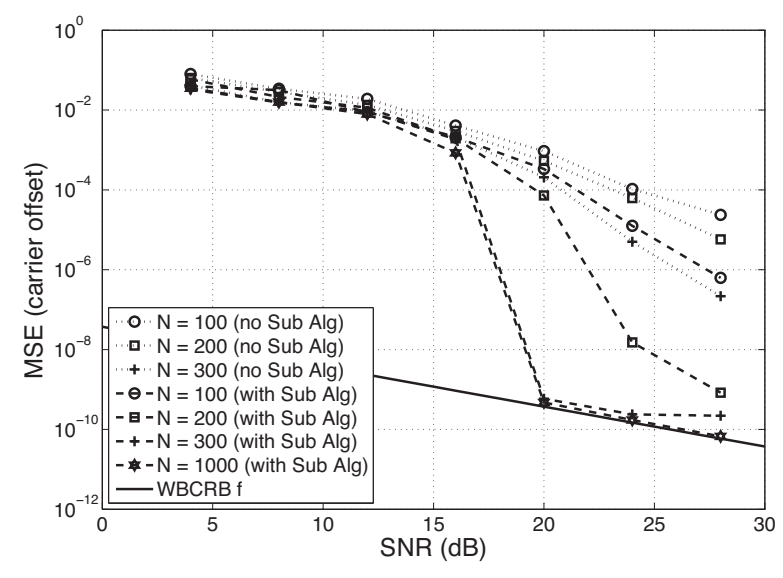

Fig. 4. MSE of carrier offset estimation as a function of SNR (dB), for different number of particles $N=100,200,300$ and 1000 .

algorithm, as a function of SNR. We can see that the BER improves with increasing the number of particles and using the proposed resampling guidelines. In addition, the slope of the BER curves for $N=200$ and 300 particles approaches that of the theoretical BPSK BER curve (plotted for perfect synchronization case), which confirms the correct working of the proposed algorithm. Note that the BER results in Fig. 5 correspond to the worst case scenario with timing and carrier offsets uniformly distributed in the full acquisition range.

\section{B. Comparison with Existing Non-Particle Filter Based Solu- tion}

The simulation results for the non-particle filter based solution in [2] achieve the theoretical BER curve for AWGN channel after $\mathrm{SNR}=6 \mathrm{~dB}$ approximately. Our result for $N=300$ particles, as shown in Fig. 5 , is $10 \mathrm{~dB}$ from the theoretical BER curve. The reason behind this performance gap is the limited range of carrier offset acquisition, $(-0.05,0.05)$ and oversampling, as exploited in [2]. As shown in Fig. 6, the BER of our particle filter based solution achieves the theoretical lower bound at $\mathrm{SNR}=10 \mathrm{~dB}$, given oversampling is used, $(Q=4)$ and the carrier offset is uniformly distributed in the range $(-0.05,0.05)$. With oversampling, the particle filtering algorithm is run on each sample of the oversampled signal before matched filtering. The probability mass function for each particle is accumulated for $Q$ adjacent samples and then new symbol is drawn after every $Q$ samples from the accumulated probability mass function. The advantage of our particle filter based solution, compared to [2], is that it can work for full range acquisition of carrier offset estimation as shown in Fig. 5.

\section{CONCLUSIONS}

In this paper, we have studied the problem of jointly estimating the timing and carrier offset and transmitted data in AWGN channels using particle filters based on the sequential importance sampling methodology. We have proposed novel resampling guidelines to deal with the degeneracy problem and fine tuning the estimated values. While the resampling guidelines in Sub-Algorithm-I and III are specific for our 


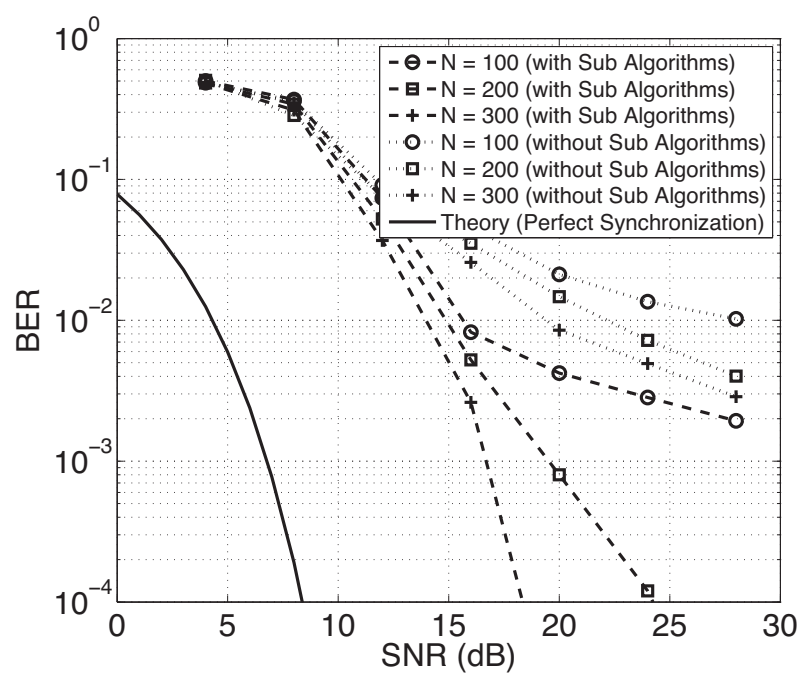

Fig. 5. BER of the particle filter receiver as a function of $\mathrm{SNR}(\mathrm{dB})$, for different number of particles $N=100,200$ and 300 with no oversampling and full range acquisition for timing and carrier offsets.

estimation problem, Sub-Algorithm-II can, in principle, be applied to deal with the degeneracy issue in static parameter estimation using particle filters in any other problem. Further, we have derived the WBCRB, which is an accurate lower bound for joint estimation of timing and carrier offsets. The performance of the proposed blind receiver is studied using computer simulations. Our simulation results illustrate that the performance of the proposed receiver, incorporating the new resampling guidelines, is close to optimal at mid-to-high SNR values, both in terms of MSE of timing and frequency offsets and BER. The advantage of our particle filter algorithm, compared to existing techniques, is that it can work for the full range acquisition of both timing and carrier offsets.

\section{APPENDIX A}

DERIVATION OF (12)

Applying Bayes theorem and laws of conditional probability, we can further simplify (11) as

$$
\begin{aligned}
& p\left(\mathbf{x}_{0: k}^{(i)}, f_{0: k}^{(i)}, \tau_{0: k}^{(i)} \mid z_{0: k}\right) \\
& =C_{k} \times p\left(\mathbf{x}_{0: k}^{(i)}, f_{0: k}^{(i)}, \tau_{0: k}^{(i)}, z_{k} \mid z_{0: k-1}\right) \\
& =C_{k} \times p\left(\mathbf{x}_{k}^{(i)}, \mathbf{x}_{0: k-1}^{(i)}, f_{0: k}^{(i)}, \tau_{0: k}^{(i)}, z_{k} \mid z_{0: k-1}\right) \\
& \stackrel{(a)}{=} C_{k} \times p\left(\mathbf{x}_{k}^{(i)} \mid \mathbf{x}_{0: k-1}^{(i)}, f_{0: k}^{(i)}, \tau_{0: k}^{(i)}, z_{k}, z_{0: k-1}\right) \\
& \times p\left(\mathbf{x}_{0: k-1}^{(i)}, f_{0: k}^{(i)}, \tau_{0: k}^{(i)}, z_{k} \mid z_{0: k-1}\right) \\
& =C_{k} \times \underbrace{p\left(\mathbf{x}_{k}^{(i)} \mid \mathbf{x}_{0: k-1}^{(i)}, f_{0: k}^{(i)}, \tau_{0: k}^{(i)}, z_{0: k)}\right)}_{1 \text { st term in (12) }} \\
& \times \underbrace{p\left(\mathbf{x}_{0: k-1}^{(i)}, f_{0: k}^{(i)}, \tau_{0: k}^{(i)}, z_{k} \mid z_{0: k-1}\right)}_{\text {Term A }},
\end{aligned}
$$

where $(a)$ is obtained using $p(A, B \mid C)=p(A \mid B, C) p(B \mid C)$.

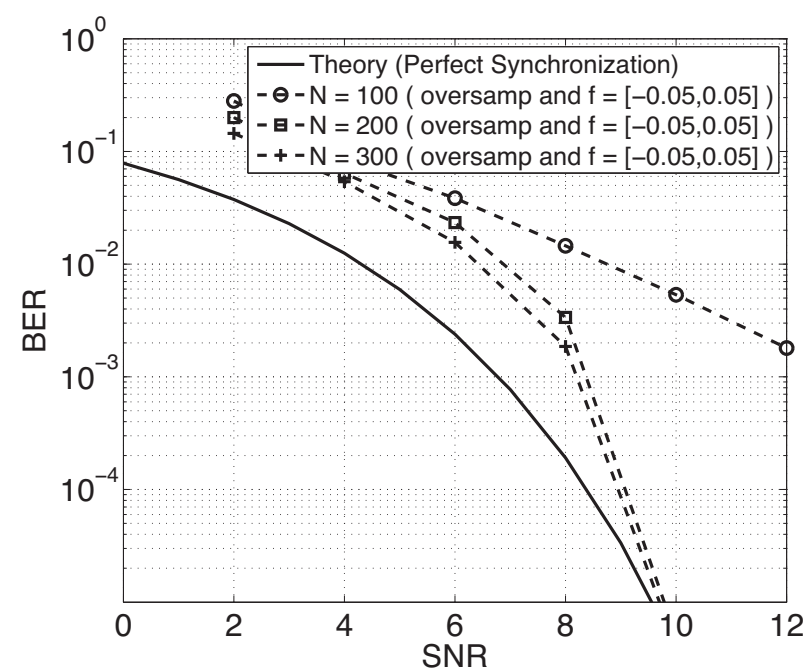

Fig. 6. BER of the particle filter receiver as a function of SNR (dB), for different number of particles $N=100,200$ and 300 with oversampling factor $Q=4$ and carrier offset acquisition range $(-0.05,0.05)$.

Decomposing Term A in (36), we have

$$
\begin{aligned}
p\left(\mathbf{x}_{0: k-1}^{(i)}, f_{0: k}^{(i)}, \tau_{0: k}^{(i)}, z_{k} \mid z_{0: k-1}\right) & \\
=p\left(z_{k} \mid \mathbf{x}_{0: k-1}^{(i)},\right. & \left.f_{0: k}^{(i)}, \tau_{0: k}^{(i)}, z_{0: k-1}\right) \\
& \times \underbrace{p\left(\mathbf{x}_{0: k-1}^{(i)}, f_{0: k}^{(i)}, \tau_{0: k}^{(i)} \mid z_{0: k-1}\right)}_{\text {Term B }} .
\end{aligned}
$$

Since the current observation in the particle filter depends only on the current state and independent of the previous states given the current state, we have $p\left(z_{k} \mid \mathbf{x}_{0: k-1}^{(i)}, f_{0: k}^{(i)}, \tau_{0: k}^{(i)}, z_{0: k-1}\right)=\underbrace{p\left(z_{k} \mid \mathbf{x}_{k-1}^{(i)}, f_{k}^{(i)}, \tau_{k}^{(i)}\right)}_{\text {2nd term in (12) }}$. De-

composing Term B in (37), we have

$$
\begin{aligned}
p\left(\mathbf{x}_{0: k-1}^{(i)}, f_{0: k}^{(i)}, \tau_{0: k}^{(i)} \mid z_{0: k-1}\right) & p\left(f_{k}^{(i)}, \tau_{k}^{(i)}, \mathbf{x}_{0: k-1}^{(i)}, f_{0: k-1}^{(i)}, \tau_{0: k}^{(i)} \mid z_{0: k-1}\right) \\
= & \underbrace{p\left(f_{k}, \tau_{k} \mid \mathbf{x}_{0: k-1}^{(i)}, f_{0: k-1}^{(i)}, \tau_{0: k-1}^{(i)}, z_{0: k-1}\right)}_{\text {Term C }} \\
& \times \underbrace{p\left(\mathbf{x}_{0: k-1}^{(i)}, f_{0: k-1}^{(i)}, \tau_{0: k-1}^{(i)} \mid z_{0: k-1}\right)}_{3 \text { rd term in (12) }} .
\end{aligned}
$$

Finally since $f_{k}^{(i)}$ and $\tau_{k}^{(i)}$ are independent variables, given $z_{k}$ is not observed, we can decompose Term $\mathrm{C}$ in (38) as

$$
\begin{aligned}
p\left(f_{k}^{(i)}, \tau_{k}^{(i)} \mid \mathbf{x}_{0: k-1}^{(i)}, f_{0: k-1}^{(i)}, \tau_{0: k-1}^{(i)}, z_{0: k-1}\right) & \underbrace{p\left(f_{k}^{(i)} \mid \mathbf{x}_{0: k-1}^{(i)}, f_{0: k-1}^{(i)}, \tau_{0: k-1}^{(i)}, z_{0: k-1}\right)}_{\text {4th term in (12) }} \\
& \times \underbrace{p\left(\tau_{k}^{(i)} \mid \mathbf{x}_{0: k-1}^{(i)}, f_{0: k-1}^{(i)}, \tau_{0: k-1}^{(i)}, z_{0: k-1}\right)}_{\text {5th term in (12) }} .
\end{aligned}
$$

Thus we arrive at the result in (12).

\section{ACKNOWLEDGEMENT}

The authors would like to thank the anonymous reviewers for their valuable comments and suggestions which helped to improve the quality of this work. 


\section{REFERENCES}

[1] H. Meyr, M. Moeneclaey, and S. A. Fechtel, Digital Communication Receivers, Synchronization, Channel Estimation, and Signal Processing, J. G. Proakis, editor. Wiley Series in Telecommunications and Signal Processing, 1998.

[2] J. Gunther and T. Moon, "Burst mode synchronization of QPSK on AWGN channels using kurtosis," IEEE Trans. Commun., vol. 57, no. 8, pp. 2453-2462, Aug. 2009.

[3] A. Doucet and A. M. Johansen, "A tutorial on particle filtering and smoothing: fifteen years later," in Handbook of Nonlinear Filtering, D. Crisan and B. Rozovsky, editors. Oxford University Press, 2011.

[4] P. M. Djuric, J. Kotecha, J. Zhang, Y. Huang, T. Ghirmai, M. F. Bugallo, and J. Miguez, "Particle filtering," IEEE Signal Process. Mag., vol. 20, no. 5, pp. 19-38, Sep. 2003.

[5] F. Daum, "Nonlinear filters: beyond the Kalman filter," IEEE Aerosp. Electron. Syst. Mag., vol. 20, no. 8, pp. 57-69, Aug. 2005.

[6] L. Mihaylova, D. A. S. Honary, D. R. Bull, and C. N. C. B. Ristic, "Mobility tracking in cellular networks using particle filtering," IEEE Trans. Wireless Commun., vol. 6, no. 10, pp. 3589-3599, Oct. 2007.

[7] O. Ozdemir, R. Niu, and P. K. Varshney, "Tracking in wireless sensor networks using particle filtering: physical layer considerations," IEEE Trans. Signal Process., vol. 57, no. 5, pp. 1987-1999, May 2009.

[8] M. M. Olama, S. M. Djouadi, I. G. Papageorgiou, and C. D. Charalambous, "Position and velocity tracking in mobile networks using particle and Kalman filtering with comparison," IEEE Trans. Veh. Technol., vol. 57, no. 2, pp. 1001-1010, Mar. 2008.

[9] J. Lim and D. Hong, "Frequency-selective and nonlinear channel estimation with unknown noise statistics," IEEE Commun. Lett., vol. 14, no. 3, pp. 245-247, Mar. 2010.

[10] D. Angelosante, E. Biglieri, and M. Lops, "Sequential estimation of multipath MIMO-OFDM channels," IEEE Trans. Signal Process., vol. 57, no. 8, pp. 3167-3181, Aug. 2009.

[11] A. Guimaraes, B. Ait-El-Fquih, and F. Desbouvries, "A fixed-lag particle smoother for blind SISO equalization of time-varying channels," IEEE Trans. Wireless Commun., vol. 9, no. 2, pp. 512-516, Feb. 2010.

[12] C. J. Bordin and M. G. S. Bruno, "Particle filters for joint blind equalization and decoding in frequency-selective channels," IEEE Trans. Signal Process., vol. 56, no. 6, pp. 2395-2405, June 2008.

[13] T. Ghirmai, N. F. Bugallo, J. Miguez, and P. M. Djuri, "A sequential Monte Carlo method for adaptive blind timing estimation and data detection," IEEE Trans. Signal Process., vol. 53, no. 8, pp. 2855-2865, Aug. 2005.

[14] F. Z. Merli, X. Wang, and G. M. Vitetta, "A Bayesian multiuser detection algorithm for MIMO-ODFM systems affected by multipath fading, carrier frequency offset, and phase noise," IEEE J. Sel. Areas Commun., vol. 26, no. 3, pp. 506-516, Apr. 2008.

[15] Z. Jing, L. Han-wen, and J. Rong-hong, "A particle filter for frequency synchronization in MIMO-OFDM systems," in Proc. 2009 WiCom.

[16] E. Serpedin and Q. M. Chaudhari, Synchronization in Wireless Sensor Networks: Parameter Estimation, Performnace Benchmarks and Protocols. Cambridge University Press, 2011.

[17] O. Tipmongkolsilp, S. Zaghloul, and A. Jukan, "The evolution of cellular backhaul technologies: current issues and future trends," IEEE Commun. Surveys Tuts., no. 99, pp. 1-17, 2010.

[18] N. Kantas, A. Doucet, S. Singh, and J. Maciejowski, "An overview of sequential Monte Carlo methods for parameter estimation in general state-space models," in Proc. 2009 IFAC Symp. Syst. Identification.

[19] C. Andrieu, A. Doucet, S. Singh, and V. B. Tadic, "Particle methods for change detection, system identification, and control," Proc. IEEE, vol. 92, no. 3, pp. 423-438, Mar. 2004.

[20] A. Doucet and V. B. Tadic, "Parameter estimation in general state-space models using particle methods," Ann. Inst. Statist. Math., vol. 55, no. 2, pp. 409-422, 2003.

[21] J. Liu and M. West, Combined Parameter and State Estimation in Simulation-based Filtering, A. Doucet, N. de Freitas, and N. Gordon, editors. Springer, 2001.

[22] G. Storvik, "Particle filters for state space models with the presence of unknown static parameters," IEEE Trans. Signal Process., vol. 50, no. 2, pp. 281-289, Feb. 2002.

[23] F. Campillo and V. Rossi, "Convolution particle filter for parameter estimation in general state-space models," IEEE Trans. Aerosp. Electron. Syst., vol. 45, no. 3, pp. 1063-1072, 2009.

[24] X. Yang, K. Xing, K. Shi, and Q. Pan, "Joint state and parameter estimation in particle filtering and stochastic optimization," J. Control Theory Appl., vol. 6, no. 2, pp. 215-220, 2008.
[25] A. Giremus, E. Grivel, J. Grolleau, and M. Najim, "A Rao-Blackwellized particle filter for joint channel/symbol estimation in MC-DS-CDMA systems," IEEE Trans. Commun., vol. 58, no. 8, pp. 2292-2304, Aug. 2010.

[26] A. A. Nasir, S. Durrani, and R. A. Kennedy, "Modified constant modulus algorithm for joint blind equalization and synchronization," in Proc. 2010 IEEE AusCTW.

[27] K. N. Oh and Y. O. Chin, "Modified constant modulus algorithm: blind equalization and carrier phase recovery algorithm," in Proc. 1995 IEEE ICC, vol. 1, pp. 498-502.

[28] T. Fusco and M. Tanda, "Blind synchronization for OFDM systems in multipath channels," IEEE Trans. Wireless Commun., vol. 3, no. 3, pp. 1340-1348, Mar. 2009.

[29] R. Mo, Y. H. Chew, T. T. Tjhung, and C. C. Ko, "A new blind joint timing and frequency offset estimator for OFDM systems over multipath fading channels," IEEE Trans. Veh. Technol., vol. 57, no. 5, pp. 29472957, Sep. 2008.

[30] J. Riba and G. Vazquez, "Bayesian recursive estimation of frequency and timing exploiting the cyclostationarity property," Signal Process., vol. 40, pp. 21-37, Jan. 1994.

[31] H. L. V. Trees and K. L. Bell, Bayesian Bounds for Parameter Estimation and Nonlinear Filtering / Tracking, M. E. El-Hawary, editor. Wiley Interscience, 2007.

[32] X. Li, Y. C. Wu, and E. Serpedin, "Timing synchronization in decode and forward cooperative communication systems," IEEE Trans. Signal Process., vol. 57, no. 4, pp. 1444-1455, Apr. 2009.

[33] Y. Wang, K. Shi, and E. Serpedin, "Non data-aided feedforward carrier frequency offset estimators for QAM constellations: a nonlinear leastsquares approach," EURASIP J. Appl. Signal Process., vol. 13, pp. $1993-$ 2001, 2004.

[34] A. Doucet, S. Godsill, and C. Andrieu, "On sequential Monte Carlo sampling methods for Bayesian filtering," Statistics Comput., vol. 10, pp. 197-208, 2000.

[35] E. Beres and R. Adve, "Blind channel estimation for orthogonal STBC in MISO systems," IEEE Trans. Veh. Technol., vol. 56, no. 4, pp. 20422050, July 2007.

[36] X. Liu, J. Kountouriotis, A. Petropulu, and K. R. Dandekar, "ALOHA with collision resolution (ALOHA-CR): theory and software defined radio implementation," IEEE Trans. Signal Process., vol. 58, no. 8, pp. 4396-4410, Aug. 2010.

[37] T. Ghirmai, "Sequential Monte Carlo method for fixed symbol timing estimation and data detection," in Proc. 2006 IEEE CISS.

[38] M. S. Arulampalam, S. Maskell, N. Gordon, and T. Clapp, "A tutorial on particle filters for online nonlinear/non-gaussian Bayesian tracking," IEEE Trans. Signal Process., vol. 50, no. 2, pp. 174-188, Feb. 2002.

[39] S. M. Kay, Fundamentals of Statistical Signal Processing: Estimation Theory. Prentice Hall, 1993.

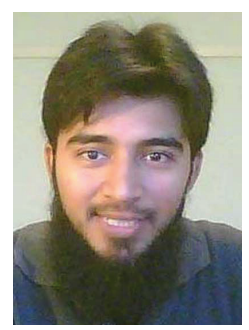

Ali A. Nasir received his B.Sc. (1st class hons.) degree in Electrical Engineering from the University of Engineering and Technology (UET), Lahore, Pakistan in 2007. He is currently pursuing his Ph.D. degree from the Research School of Engineering, the Australian National University, Canberra, Australia. Prior to joining ANU, he worked as a Lecturer in UET for nine months and then as a Design Engineer in the Centre for Advanced Research in Engineering (CARE), Islamabad, Pakistan for a year. He was a research visitor in Chalmers University of Technology, Gothenburg, Sweden for six months from April till October 2011. Ali was awarded a University Gold Medal for outstanding performance during the final year of his undergraduate studies. He is a recipient of an ANU International Ph.D. scholarship for the duration of his Ph.D. He was also awarded an ANU Vice Chancellor's Higher Degree Research (HDR) travel grant in 2011. His research interests are in the area of synchronization and channel estimation in cooperative communication, MIMO and OFDM systems. 


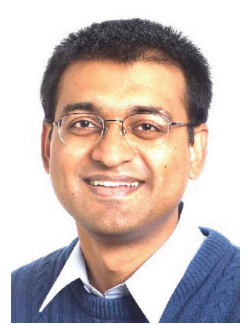

Salman Durrani (S'00-M'05-SM'10) received the B.Sc. (1st class honours) degree in Electrical Engineering from the University of Engineering \& Technology, Lahore, Pakistan in 2000. He received the $\mathrm{PhD}$ degree in Electrical Engineering from the University of Queensland, Brisbane, Australia in Dec. 2004. He has been with the Australian National University, Canberra, Australia, since 2005, where he is currently Senior Lecturer in the Research School of Engineering, College of Engineering \& Computer Science. His current research interests are in wireless communications and signal processing, including synchronization in cooperative communication systems, connectivity of ad-hoc networks and vehicular networks and signal processing on the unit sphere. He serves as a Technical Program Committee Member for international conferences such as ICC '12, PIMRC '12 and AusCTW '12. He was a recipient of an International Postgraduate Research Scholarship from the Australian
Commonwealth during 2001-2004. He was awarded a University Gold Medal during his undergraduate studies. He is a Member of Institution of Engineers, Australia and a Senior Member of IEEE.

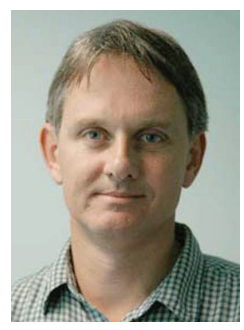

Rodney A. Kennedy (S'86-M'88-SM'01-F'05) received the B.E. degree from the University of New South Wales, Sydney, Australia, the M.E. degree from the University of Newcastle, and the Ph.D. degree from the Australian National University, Canberra. He is currently a Professor in the Research School of Engineering, Australian National University. He is a Fellow of the IEEE. His research interests include digital signal processing, digital and wireless communications, and acoustical signal processing. 